\title{
Reducing the overuse of $\beta$ hCG measurements in the emergency gynaecology clinic
}

Lucy Frost

Royal Berkshire Hospital, United Kingdom

\begin{abstract}
Serial $\beta$ hCG testing can be a helpful tool in deciding how to manage pregnancy of unknown location. Its use in emergency gynaecology clinics can prevent unnecessary admission and intervention. However, despite NICE Guidelines on when it is safe to opt for conservative management, it was identified that there was a problem with over-testing of $\beta$ hCG when patients could be discharged with instructions to repeat a urinary pregnancy test in two weeks. Two PDSA cycles were undertaken to improve the awareness of NICE guidelines: the first involved formal and informal educational sessions and the second involved the inclusion of a guideline summary on the front of patients' notes when they were having serial $\beta$ hCG tests for doctors to refer to.
\end{abstract}

Case notes were reviewed for 157 women who had $\beta$ hCG tests at baseline and 48 hours. Of these, 139 were suitable for serial $\beta$ hCG testing, and 83 of these were suitable for discharge after 48 hours. Of the 83 patients that were eligible for discharge, there were 31 unnecessary $\beta \mathrm{hCG}$ tests done, 23 of which were prior to intervention. A significant improvement was noted, with between 4-10 unnecessary $\beta \mathrm{hCG}$ tests per fortnight prior to intervention, 0-3 following the first intervention, and 0-2 following the second. Reduction in unnecessary $\beta \mathrm{hCG}$ testing has positive implications for patients, who do not have to take unnecessary time off work, prolong an already very distressing period, and have unnecessary blood tests. There are also cost and time saving implications for the hospital.

\section{Problem}

When a patient has a positive pregnancy test but no sign of an intra or extra-uterine pregnancy on transvaginal ultrasound investigation, it is described as a pregnancy of unknown location.[1] This is a common condition, affecting $8-31 \%$ of early pregnancy scans.[2] The importance of good management is clear - a pregnancy of unknown location can turn out to be a viable early pregnancy, a miscarriage, or an ectopic pregnancy. When used appropriately, serial $\beta$ hcg measurements can be a useful tool to aid management planning in these cases.[1] However, over-testing or failing to act on significant results can result in unnecessary delays in either discharge from clinic or further investigation. It was identified by medical and nursing staff in the emergency gynaecology clinic at the Royal Berkshire Hospital that frequent over-testing of $\beta$ hcg has been noted, despite NICE criteria for discharge being met.

\section{Background}

The emergency gynaecology clinic sees many patients with bleeding and pain in early pregnancy. Without a previous scan confirming an intrauterine pregnancy then these patients must be treated as having a pregnancy of unknown location. Serial $\beta \mathrm{hCG}$ measurements are a common method of determining which patients need to be treated as high risk of an ectopic pregnancy, and which patients are safe to treat conservatively. NICE Guidelines CG 154 state that if the 48 hour $\beta$ hCG measurement is $50 \%$ lower than the initial test, with an empty uterus on scan, then the patient can be discharged from clinic to do a urinary pregnancy test in two weeks.[1] The patients with positive pregnancy tests at that stage would then need to be followed up at the clinic, however evidence suggests that the vast majority will have spontaneously resolved by this point and this is a safe course of action.[3-6]

\section{Baseline measurement}

The outcome measure used was the number of unnecessary $\beta \mathrm{hCG}$ tests done per fortnight in the emergency gynaecology clinic. To be included, the patient needed to be appropriate for serial $\beta \mathrm{hCG}$ management. This meant they needed to have a single ultrasound scan showing an empty uterus, with bleeding and/or pain in the first trimester.[1] As a baseline, this was measured over six weeks.

\section{Design}

It was decided that the key area to target was how doctors make decisions about what to do when reviewing baseline and 48 hour $\beta h C G$ results. Through discussions with various members of the team, observation of decision making, and reviewing previous management decisions it became clear that there are vast differences between how different doctors make decisions, and even about how the same doctor may treat patients with similar $\beta h C G$ results. Discussion with the doctors emphasised that this was largely due to a lack of clear guidance on how decisions should be made and therefore lack of confidence.

Despite the presence of NICE guidelines on this topic, many doctors were unaware of how these guidelines applied to what they were doing. We proposed to build on an existing system: as it currently works, when a patient is put on a serial $\beta \mathrm{hCG}$ 
management plan a front sheet is added to the front of each patient's notes. On this front sheet, a member of nursing staff records each $\beta$ hCG result so all can be reviewed at the same time. We decided that this front sheet could also inform and encourage people to follow the guidelines as they only made decisions through looking at this sheet. This would also ensure sustainability of the intervention, as the change would be there permanently as long as the system of front sheets remained the same.

\section{Strategy}

PDSA cycle 1: Educational sessions were undertaken with doctors and nursing staff to assess whether knowledge of current guidelines would be sufficient to change practice. These education sessions took the form of formal teaching sessions and informal, educational discussions with stakeholders. This covered provision of information on current guidelines, basic evidence review, and some worked examples, and was varied according to what was required by each clinician. Discussions on concerns and motivations for current practice were also included to ensure that we could address these issues fully. Both doctors and nurses were very receptive to this, and there was an immediate decline in unnecessary $\beta$ hCG tests. We therefore decided to continue to implement a more sustainable intervention to ensure awareness of guidelines.

PDSA cycle 2: "50\% off" reminder cards were added to the front sheet on all patients' notes who were having serial $\beta \mathrm{hCG}$ management. These summarised key messages from the NICE guidelines, empowering doctors to make the decision to discharge patients if they had a 50\% BhCG drop after 48 hours, with instructions to repeat a urinary pregnancy test after two weeks. It also served as a way that nursing staff would be able to question doctors decisions if this decision was not made.

See supplementary file: ds6611.docx - "PDSA Cycles"

\section{Post-measurement}

The number of unnecessary $\beta \mathrm{hCG}$ results were measured fortnightly over 20 weeks. Over that time period, 157 women had $\beta h C G$ measured at baseline and 48 hours. Their notes and ultrasound results were reviewed to assess whether they were appropriate for serial $\beta \mathrm{hCG}$ management, and if so if they were appropriate for discharge after their 48 hour $\beta$ hCG. Of these, 139 were appropriate for serial $\beta$ hCG management and 83 patients were appropriate for discharge after their 48 hour $\beta$ hCG, according to NICE guidelines. The reason that 18 patients were not appropriate for serial $\beta \mathrm{hCG}$ management was because they did not have an ultrasound scan. Of the 83 patients that were eligible for discharge, there were 31 unnecessary $\beta$ hCG tests done. 23 of these unnecessary $\beta \mathrm{hCG}$ tests were done pre-intervention.

Following intervention, there was a marked decrease in the number of unnecessary $\beta \mathrm{hCG}$ tests done as soon as the education campaign began (Range pre-intervention $=4-10$; Range postintervention $1=0$ - 3; Range post-intervention $2=0$ - 2). Additionally, there was a reduction in the variability between measurements. Although the number of unnecessary $\beta$ hCG tests did not continue to drop significantly with the second PDSA cycle, the change was sustained.

The fortnightly data collection frequency was chosen due to the fact notes are stored in the department for two weeks so it allowed easy evaluation of all the notes, whilst allowing sufficient sample size to analyse data.

See supplementary file: ds6612.pptx - "Run chart"

\section{Lessons and limitations}

One challenge with the intervention was that the ' $50 \%$ off' cards were an additional thing to attach the the front of the notes, in addition to the front sheet. As the nursing staff were engaged with the project this was done in all cases, but I can foresee that this may not continue or may not have worked in a different setting. The reason this decision was made was because we hoped to implement trust guidelines on the subject and wanted these to be ratified before making any changes to the front sheet itself to avoid any confusion. This ratification process took longer than anticipated and therefore this change has not been made yet. However, this would be the next step to rolling out the project and would encourage sustainability of the project.

Another issue was planning around the changeover of medical staff. The initial part of the intervention was a time-intensive educational campaign on the guidelines. It is unclear whether the ' $50 \%$ off' card intervention would have been successful without this campaign, therefore it is difficult to know what will happen when the doctors change over. Ideally we would have continued data collection over this period but this was not possible due to the authors' changeover. The interventions were handed over but the new project leaders were unable to continue with fortnightly data collection. Earlier consideration of the capacity of those taking over the project may have allowed different design to accommodate this or involve more people with the data collection.

\section{Conclusion}

Over the course of 20 weeks, there was a significant reduction in the number of unnecessary $\beta \mathrm{hCG}$ measurements. There was also significant positive feedback from staff who commented on a subjective improvement in action plans based on guidelines, the potential for cost and quality of life benefits, and reassurance in having a protocol to use. They were all excited about the idea of trust guideline implementation, which is currently awaiting ratification at clinical governance meeting. Although we have not yet reached $0 \%$ unnecessary $\beta h C G$ tests in serial $\beta$ hCG management plans, it is much better than previously and more patients are being managed in line with NICE guidance. This will also have benefits in terms of patients not having to take unnecessary time off work, reduction in number of returns to hospital (which can be distressing following a miscarriage), and fewer unnecessary, painful blood tests. For the hospital there will be benefits in terms of saved staff time costs, saved appointments that can be used for other patients, 


\section{BMJ Quality Improvement Reports}

and saved costs of taking and analysing unnecessary blood tests.

ethical approval.

Further steps will need to focus around how we can ensure the number of unnecessary $\beta$ hCG tests remains low around periods of doctor changeover. This is likely to require further empowerment of the nursing and healthcare assistant staff, and more permanent changes to the front sheet. Additionally, it might be helpful to involve staff from $A+E$ and GP surgeries, who may be involved in taking initial $\beta \mathrm{hCG}$ measurements and referral to the emergency gynaecology clinic.

\section{References}

1 National Institute for Health and Care Excellence. Ectopic pregnancy and miscarriage: diagnosis and initial management. NICE guideline (CG154); 2012.

2 Sagili $\mathrm{H}$, Mohamed K. Pregnancy of unknown location: an evidence-based approach to management. The Obstetrician \& Gynaecologist 2008; 10: 224-230.

3 Hahlin M, Thorburn J, Bryman I. The expectant management of early pregnancies of uncertain site. Hum Reprod 1995; 10: 1223-1227.

4 Banerjee S, Aslam N, Zosmer N, Woelfer B, Jurkovic D. The expectant management of women with pregnancies of unknown location. Ultrasound Obstet Gynecol 1999; 14: 231-236.

5 Banerjee S, Aslam N, Woelfer B, Lawrence A, Elson J, Jurkovic D. Expectant management of early pregnancies of unknown location: a prospective evaluation of methods to predict spontaneous resolution of pregnancy. BJOG 2001; 108: 158-163.

6 Condous G, Lu C, Van Huffel S, Timmerman D, Bourne T. Human chorionic gonadotrophin and progesterone levels for the investigation of pregnancies of unknown location. Int J Gynaecol Obstet 2004; 86: 351-357.

\section{Declaration of interests}

Nothing to declare

\section{Acknowledgements}

Aye Aye, William Kuteesa, Jayne Waite

\section{Ethical approval}

Ethics approval was not sought as this work was quality improvement work. There was no contact with patients and no patient identifiable data was captured or stored during the process. Further, the only encouraged change in practice was to bring practice in line with NICE guidelines and there were no experimental changes encouraged. Therefore, local policy at the Royal Berkshire Hospital NHS Foundation Trust did not require 